\title{
Perovskite-oxide based hyperbolic metamaterials
}

Mohamed Bouras, Dong Han, Sébastien Cueff, Romain Bachelet and Guillaume SaintGirons.

\section{Supporting information}
11 pages
8 figures
3 tables 


\section{Structural characterizations of the LSTO layers and LSTO/STO superlattices:}

High-resolution X-ray diffraction (XRD) experiments were carried out using a Rigaku Smartlab diffractometer equipped with a high brilliance rotating anode and a Ge (220) two-bounces monochromator. Fig.SI.1 displays wide-range out-of-plane radial scans recorded on the three LSTO layers (samples L-5\%, L-10\% and L-20\%). These scans only present 001 reflexions, attesting for the epitaxial growth of LSTO layers on the STO substrates. Zooms around the (002) reflexion regions for the three samples are shown in Fig.SI.2(a), and rocking curves recorded around these reflexions are shown in Fig.SI.2(b).

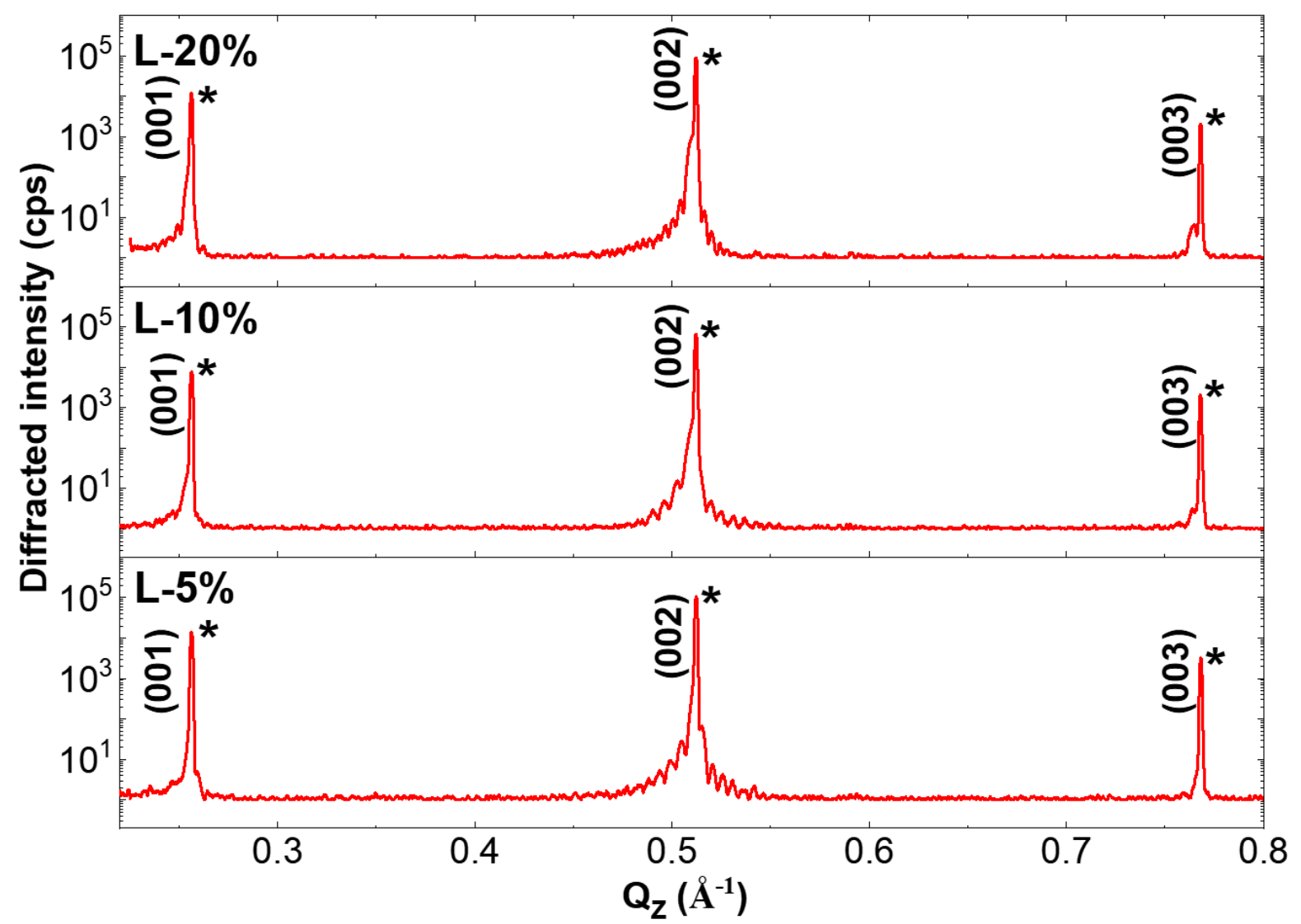

Fig.S1: Out-of-plane XRD scans recorded on samples L-5\%, L-10\% and L-20\%. * indicate the substrate reflexions, and $\mathrm{Q}_{\mathrm{z}}$ is the out-of-plane diffraction vector. 
(a)

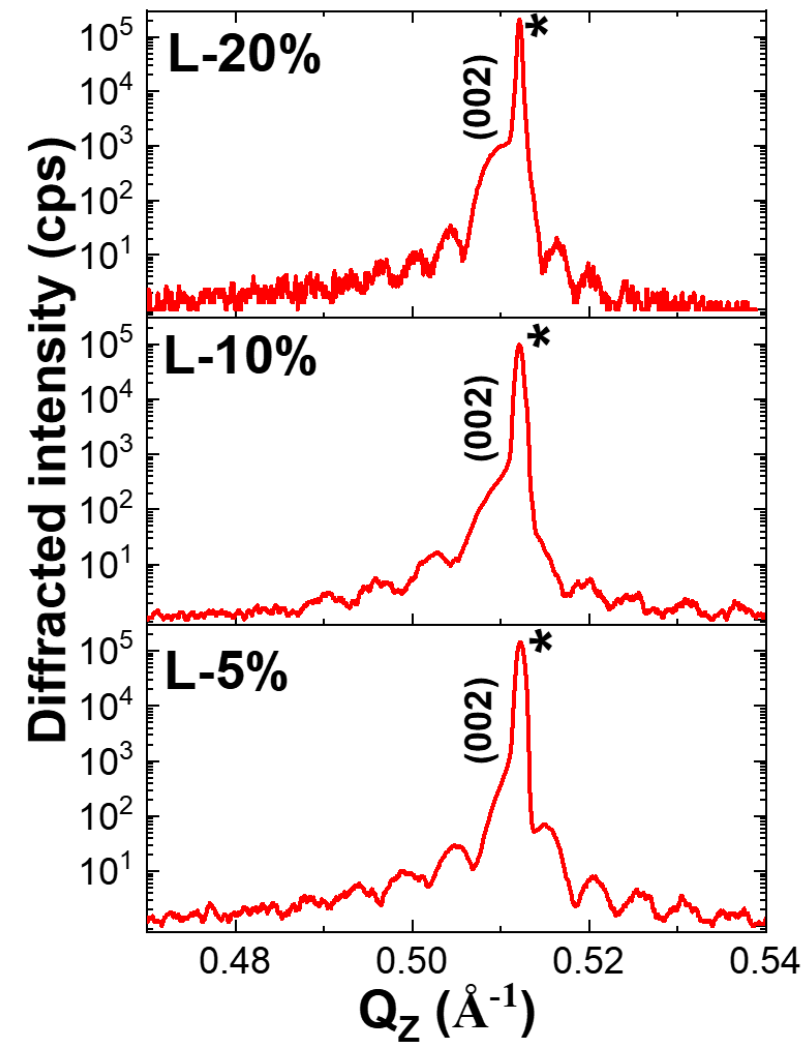

(b)

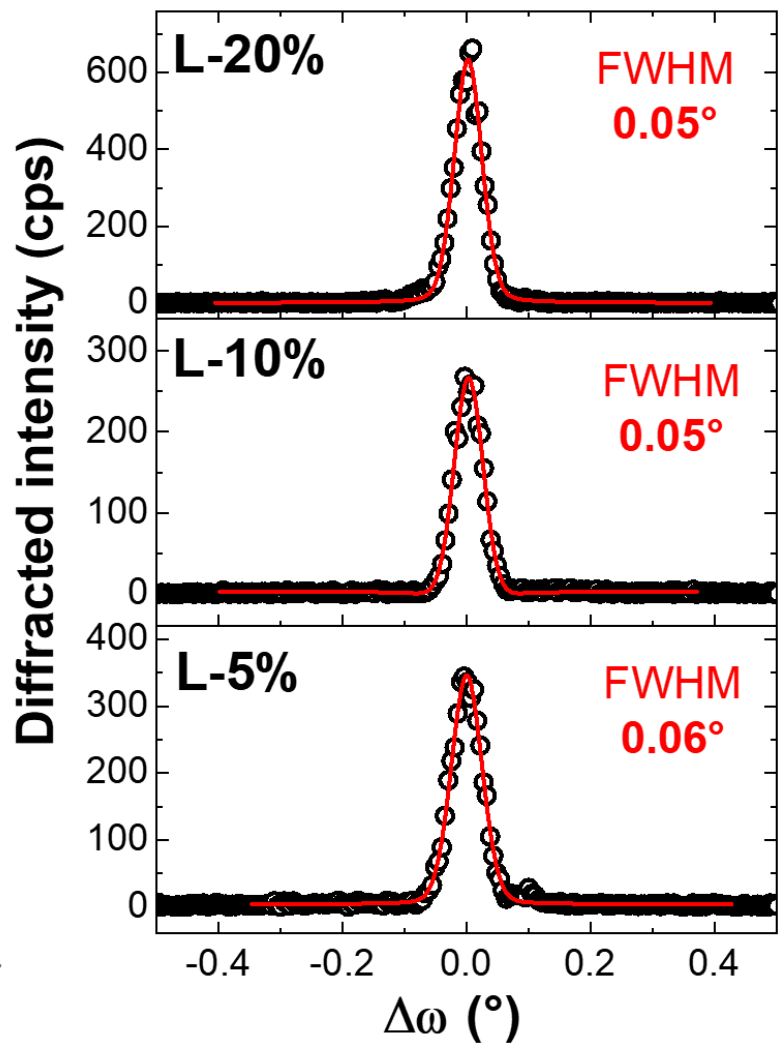

Fig.S2: Out-of-plane XRD scans recorded around the (002) reflexions for samples L-5\%, L-10\% and L$20 \%$. (a) : Radial scans around the (002) reflexions (* designate the substrate reflexions and $\mathrm{Q}_{z}$ the outof-plane diffraction vector). (b) : Corresponding rocking-curves.

They attest for the excellent structural quality of the LSTO layers, in keeping with state of the art results reported reported in the literature: ${ }^{1,2}$ the radial scan presents Pendellösung fringes indicating low surface roughness and excellent LSTO/STO interface abruptness, and the full width at half maximum of the rocking curve in inset is less than $0.05^{\circ}$, highlighting the excellent crystal quality of the layer. The wide range X-ray radial scans only present (001) reflexions, showing that the layers are single crystalline and epitaxially grown on the STO substrates.

X-rays reflectivity (XRR) was used to measure the thicknesses of the LSTO films (Fig.SI.3 and Tab.SI.1). 


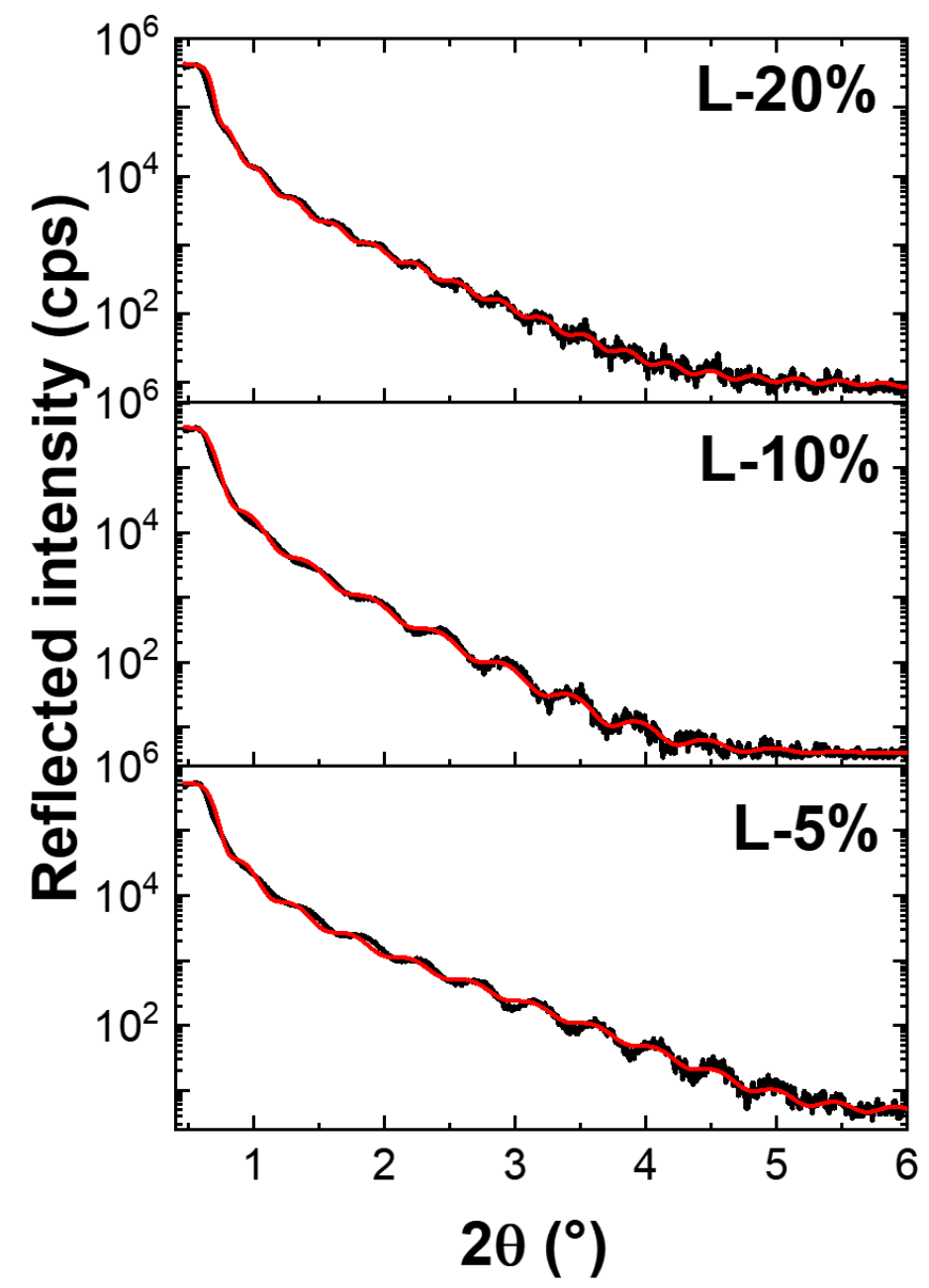

Fig.S3: X-rays reflectivity (XRR) scans recorded for samples L-5\%, L-10\% and L-20\% (black lines) and associated fits (red lines).

\begin{tabular}{|c|c|c|}
\hline Sample & Thickness $(\mathrm{nm})$ & Error $( \pm \mathrm{nm})$ \\
\hline L-5\% & 17.0 & 0.3 \\
\hline L-10\% & 15.6 & 0.3 \\
\hline L-20\% & 22.8 & 0.6 \\
\hline
\end{tabular}

Tab.S1: Thicknesses of samples L-5\%, L-10\% and L-20\% measured by XRR

Fig.SI.4 and SI.5 respectively show the XRD out-of-plane radial scans and the rocking-curves around the (002) reflexions recorded for the superlattice samples (SL-20\%-3nm, SL-20\%-5nm, SL-20\%-7nm and SL-10\%-9nm). The radial scans only displays (001) reflexions, attesting for monocrystalline and epitaxial growth. The satellite peaks were used to determine the period of $\Lambda$ the superlattices, and hence the thickness of the individual layers $(=\Lambda / 2)$ and the total thickness of the samples (number of periods $\times \Lambda$ ). The FWHM of the rocking curves are in the few $10^{-2} \circ$ range, attesting for low mosaïcities and good crystal quality. 


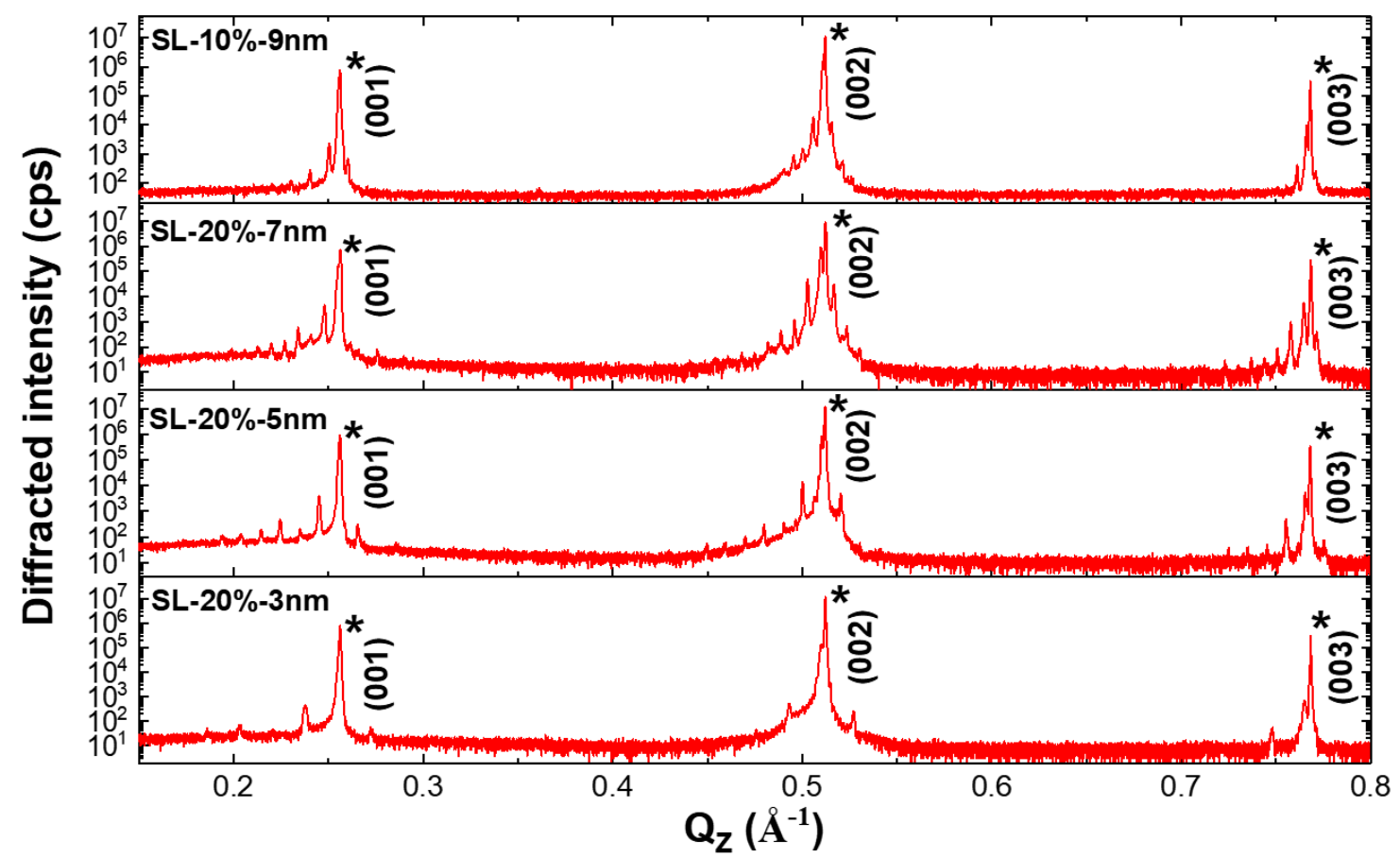

Fig.S4: Out-of-plane XRD radial scans recorded on samples SL-20\%-3nm, SL-20\%-5nm, SL-20\%-7nm and SL-10\%-9nm. * indicate the substrate reflexions, and $\mathrm{Q}_{\mathrm{z}}$ is the out-of-plane diffraction vector.
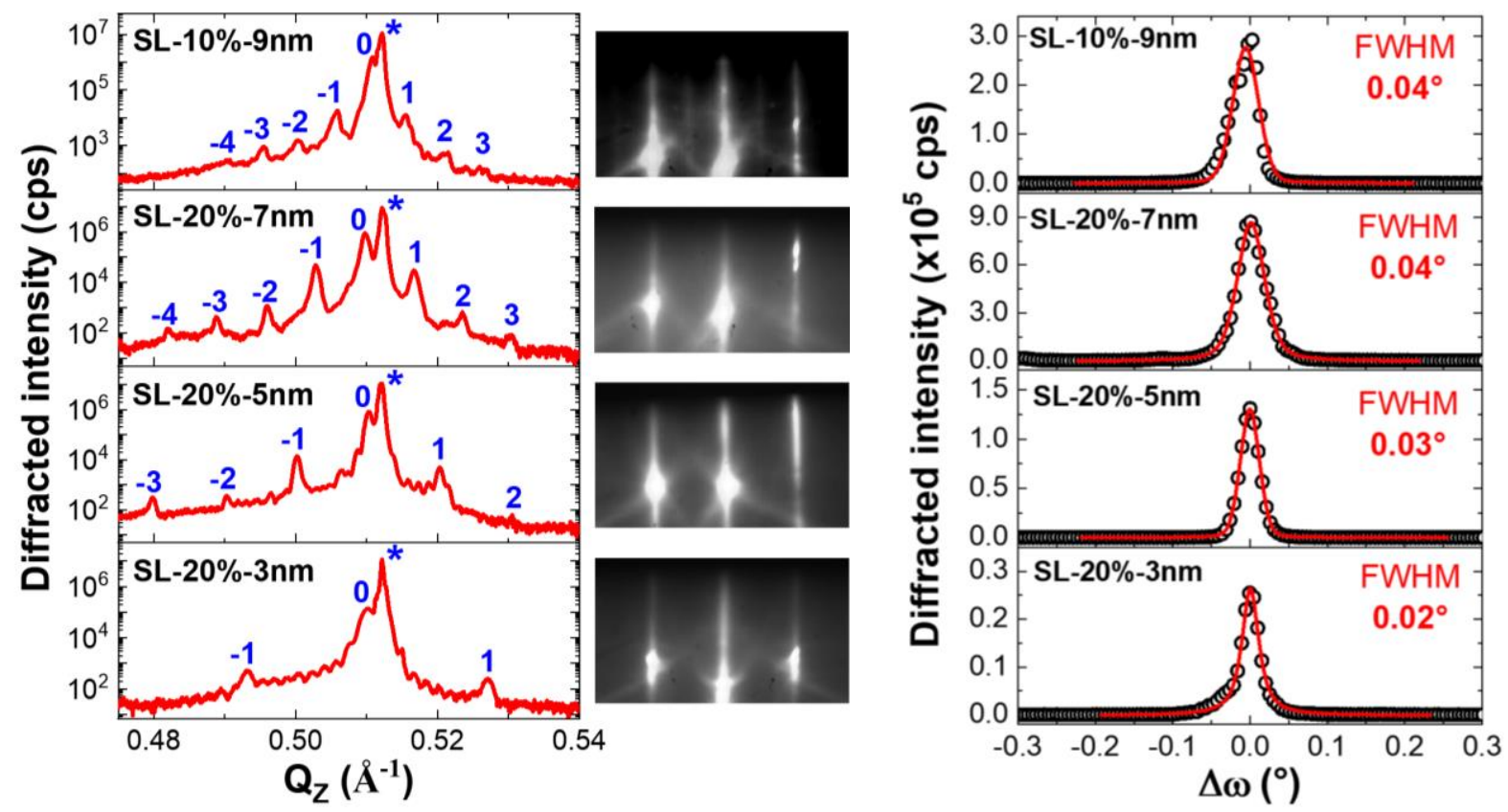

Fig.S5: Left panel : zoom around the (002) reflexions. The presence of Pendellösung fringes and of well-defined superlattice satellite reflexions (indexed using blue numbers) attests for the excellent structural quality and single-crystallinity of the samples, further confirmed by the well-contrasted streak lines of the reflection high-energy electron diffraction (RHEED) pattern recorded at the end of sample growth. Right panel : X-ray rocking curves recorded around the $0^{\text {th }}$ order satellite peaks. 


\section{Ellipsometry results:}

Spectroscopic ellipsometry was used to measure the permittivities of the LSTO layers and LSTO/STO superlattices depending on the incident beam energy E. For this purpose, we used an HORIBA-Jobin Yvon UVISEL Plus ellipsometer enabling measurements ranging from 0.59 to $4.76 \mathrm{eV}(0.26$ to $2.1 \mu \mathrm{m})$. The results presented outside this range in Fig. 3 and 4 were extrapolated using the oscillator models used to fit the ellipsometric data in the measurement range. For the LSTO layers, the ellipsometric angles $\psi$ and $\Delta$ were measured at an incident angle $\theta$ of $55^{\circ}$ while for the LSTO/STO superlattices, they were measured at 13 different incident angles regularly distributed between 55 to $81^{\circ}$ (Variable Angle Spectroscopic Ellipsometry). The $\psi(\mathrm{E})$ and $\Delta(\mathrm{E})$ functions were fitted (simultaneously for all incidence angles for the superlattice samples) using convenient oscillator models to extract the complex permittivities $\varepsilon$ and the thicknesses of the samples. In addition, a STO substrate was also analyzed using ellipsometry after an annealing in the MBE reactor simulating the growth of the LSTO layers and of the STO/LSTO superlattices (ie carried out using the same duration and the $\mathrm{O}_{2}$ partial pressure as that used for sample growth). The STO substrate dielectric function was extracted by ellipsometry, and then injected in the models used for the other samples, in a substrate-layer configuration.

Isotropic models were used for LSTO layers, and uniaxially anisotropic models were used for the superlattice samples. The discrepancy between model and experimental data was minimized numerically by minimzing a $\chi^{2}$ function :

$\chi^{2}=\sum_{E, \theta} \frac{(\text { CalcVal-MeasVal })^{2}}{\text { MeasVal }}$.

Perfect match between model and experimental data leads to $\chi^{2}=0$. Fits were performed using the HORIBA-Jobin Yvon DeltaPsi2 software.

Results for the LSTO layers (samples L-5\%, L-10\%, L-20\% and STO reference substrate)

The best fit for these samples was obtained by using an isotropic model combining one Lorentz oscillator, 3 Tauc-Lorentz oscillators and one Drude contribution (for samples containing La only).

$\varepsilon=\varepsilon_{\infty}+\varepsilon^{L}+\sum_{j=1}^{3} \varepsilon_{j}^{T L}+\varepsilon^{D}$,

where $\varepsilon_{\infty}$ is the permittivity at infinite frequency, $\varepsilon^{L}(\omega)=\frac{f \times \omega_{o}^{2}}{\omega_{o}^{2}-\omega^{2}+i \times \Gamma_{L} \times \omega}$ (with $\omega$ the radiation angular frequency, $\omega_{0}$ the peak frequency of the oscillator, $f$ the oscillator strength and $\Gamma_{\mathrm{L}}$ the broadening) is the Lorentz contribution, $\varepsilon_{D}(\omega)=-\frac{\omega_{p}^{2}}{-\omega^{2}+i \times \Gamma_{D} \omega}$ (with $\omega_{\mathrm{p}}$ the plasma frequency and $\Gamma_{\mathrm{D}}$ the broadening) is the Drude contribution and $\varepsilon^{T L}(E)=\varepsilon_{r}^{T L}(E)+$ $i \times \varepsilon_{i}^{T L}(E)$ is the Tauc-Lorentz contribution. The real and imaginary parts of the TaucLorentz contributions read ${ }^{3}$ : 
$\varepsilon_{i}^{T L}(E)=\left\{\begin{array}{c}0 \text { if } E \leq E_{g} \\ \frac{1}{E} \times \frac{A \times E_{0} \times C \times\left(E-E_{g}\right)^{2}}{\left(E^{2}-E_{0}^{2}\right)^{2}+C^{2} \times E^{2}} \text { if } E>E_{g}, \text { and }\end{array}\right.$

$\varepsilon_{r}^{T L}(E)=\frac{2}{\pi} \int_{0}^{\infty} \frac{\chi \times \varepsilon_{i}^{T L}(\chi)}{\chi^{2}-E^{2}} d \chi$,

where $\mathrm{E}_{\mathrm{g}}$ is the optical bandgap of the material, $\mathrm{E}_{0}$ is the central energy of the transition, $\mathrm{A} / \mathrm{E}_{0}$ its oscillator strength, and $\mathrm{C}$ its broadening. This model (without the Drude contribution) is very close to that reported in Ref.4 to describe STO dispersion.

Excellent agreement between experimental and calculated ellipsometric functions was obtained using this procedure, as shown in Fig.SI.6.

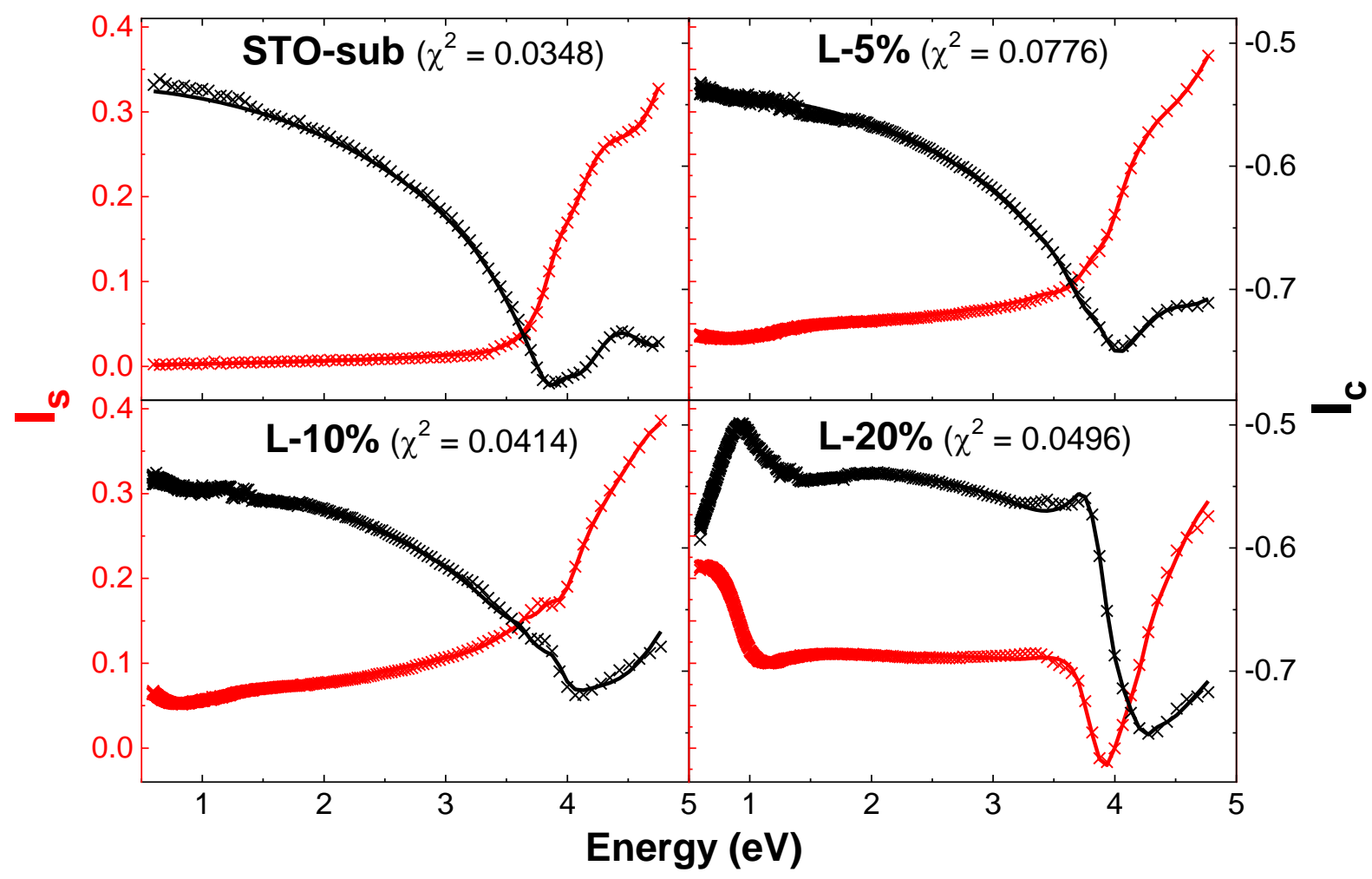

Fig.S6: Experimental (crosses) and modeled (solid lines) ellipsometric parameters for the annealed STO substrate (STO-sub) and for samples L-5\%, L-10\% and L-20\% (incident angle $\theta=55^{\circ}$ ). $\mathrm{I}_{\mathrm{s}}$ and $\mathrm{I}_{\mathrm{c}}$ are related to the ellipsometric angles $\psi$ and $\Delta$ by $I_{s}=\sin (2 \psi) \times \sin (\Delta)$ and $I_{c}=\sin (2 \psi) \times \cos (\Delta)$. Very low $\chi^{2}$ values attest for the excellent agreement between calculated and experimental ellipsometric data.

The resulting dispersions for the LSTO layers are depicted in the main text (Fig.2(a)). The associated oscillator parameters and thicknesses (measured by ellipsometry, very close to that deduced from XRR, Tab.SI.1) are gathered in Tab.SI.2. 


\begin{tabular}{|c|c|c|c|c|c|c|c|c|c|c|c|c|c|c|c|c|}
\hline \multirow[b]{2}{*}{ Sample } & \multirow[b]{2}{*}{$\varepsilon_{\infty}$} & \multirow[b]{2}{*}{$\begin{array}{c}\mathbf{E g} \\
(\mathbf{e V})\end{array}$} & \multicolumn{3}{|c|}{ Lorentz } & \multicolumn{3}{|c|}{ Tauc-Lorentz 1} & \multicolumn{3}{|c|}{ Tauc-Lorentz 2} & \multicolumn{3}{|c|}{ Tauc-Lorentz 3} & \multicolumn{2}{|c|}{ Drude } \\
\hline & & & $\mathbf{f}$ & $\begin{array}{c}\omega_{0} \\
(\mathrm{eV})\end{array}$ & $\begin{array}{c}\Gamma_{\mathbf{L}} \\
(\mathbf{e V})\end{array}$ & $\begin{array}{c}\mathrm{A} \\
(\mathrm{eV})\end{array}$ & $\begin{array}{c}\mathbf{E}_{0} \\
(\mathrm{eV})\end{array}$ & $\begin{array}{c}C \\
(\mathrm{eV})\end{array}$ & $\begin{array}{c}\mathbf{A} \\
(\mathrm{eV})\end{array}$ & $\begin{array}{c}\mathbf{E}_{0} \\
(\mathrm{eV})\end{array}$ & $\begin{array}{c}\mathrm{C} \\
(\mathrm{eV})\end{array}$ & $\begin{array}{c}\mathbf{A} \\
(\mathrm{eV})\end{array}$ & $\begin{array}{c}\mathbf{E}_{0} \\
(\mathrm{eV})\end{array}$ & $\begin{array}{c}C \\
(\mathrm{eV})\end{array}$ & $\begin{array}{c}\omega_{\mathrm{p}} \\
(\mathrm{eV})\end{array}$ & $\begin{array}{c}\Gamma_{\mathrm{D}} \\
(\mathrm{eV})\end{array}$ \\
\hline STO-sub & 1.16 & 3.22 & 1.87 & 0.71 & 0.80 & 569 & 3.85 & 1.32 & 4.03 & 4.27 & 0.17 & 0.59 & 7.51 & 0.54 & $\varnothing$ & $\varnothing$ \\
\hline L-5\% & 1.28 & 3.43 & 1.03 & 4.94 & 0.99 & 128 & 3.99 & 0.70 & 1.77 & 4.28 & 0.36 & 36.8 & 6.66 & 0 & 0.67 & 0.21 \\
\hline L-10\% & 1.33 & 3.62 & 1.46 & 5.85 & 1.37 & 82.4 & 3.65 & 0.05 & 293 & 3.94 & 0.88 & 0.55 & 4.41 & 13.0 & 1.16 & 0.38 \\
\hline L-20\% & 1.34 & 3.64 & 0.05 & 2.42 & 1.01 & 202 & 4.16 & 1.03 & 0.18 & 4.24 & 0 & 0.91 & 4.36 & 0.15 & 1.50 & 0.15 \\
\hline
\end{tabular}

\begin{tabular}{|c|c|c|}
\hline Sample & Thickness $(\mathrm{nm})$ & Error $( \pm \mathrm{nm})$ \\
\hline L-5\% & 16.9 & 0.8 \\
\hline L-10\% & 14.6 & 0.4 \\
\hline L-20\% & 22.8 & 0.2 \\
\hline
\end{tabular}

Tab.S2: Oscillator parameters and thicknesses (deduced from ellipsometry measurements) for the reference STO substrate and for sample L-5\%; L-10\% and L-20\%.

In Fig. 2 as well as Eq. 1 and 2 of the main text, $\omega_{\mathrm{p}}$ is expressed in $\operatorname{rad} \times \mathrm{s}^{-1}$ and $\Gamma_{\mathrm{D}}$ in $\mathrm{s}^{-1}$, whereas they are expressed in $\mathrm{eV}$ in Tab. SI.2. The conversion is as follows :

$\Gamma_{D}\left(s^{-1}\right)=\Gamma_{D}(e V) \times e \frac{2 \pi}{h}$, and

$\omega_{p}\left(r a d \times s^{-1}\right)=\omega_{p}(e V) \times e \frac{2 \pi}{h}$,

where $\mathrm{e}=1.6 \times 10^{-19} \mathrm{~J}$; and $\mathrm{h}$ is the Planck constant.

For the STO substrate, the model and parameters are very close to that reported in Ref. 4. Increasing the La composition leads to significant increase of the bandgap, but does not significantly modifies the oscillator structure and hence the dispersion above the bandgap (see also Fig.2(a) in the main text). Of course, La doping affects the Drude contribution (which is absent for the insulating STO substrate), as discussed in the main text.

Results for the LSTO/STO superlattices (samples SL-20\%-3nm, SL-20\%-5nm, SL-20\%-7nm and SL-10\%-9nm)

For the LSTO/STO superlattices, isotropic models as expected failed to conveniently describe the experimental results, and uniaxial anisotropic dispersions were used, with an ordinary component $\varepsilon_{\mathrm{o}}=\varepsilon_{\mathrm{r}-\mathrm{o}}+\mathrm{i} \times \varepsilon_{\mathrm{i}-\mathrm{o}}$ lying in the growth plane, and an extraordinary $\varepsilon_{\mathrm{e}}=\varepsilon_{\mathrm{r}-\mathrm{e}}+\mathrm{i} \times \varepsilon_{\mathrm{i}-\mathrm{e}}$ component lying along the growth axis (see Fig. 3 in the main text). As for the simple layer samples, a combination of Lorentz, Tauc-Lorentz and Drude contribution was used to describe ordinary and extraordinary permittivities. Following guideline was followed to choose oscillator functions liable to provide convenient fit of the ellipsometric data. In the ordinary plane, the superlattice response is expected to be an approximate average of the dielectric functions of the constitutive insulating and conductive layers, weighted by their relative thicknesses. We thus used a combination of a Drude contribution (to describe charge carrier transport in the ordinary plane) combined with Tauc-Lorentz oscillators describing the above bandgap interband transitions :

$\varepsilon_{o}=\varepsilon_{\infty}+\sum_{j} \varepsilon_{j}^{T L}+\varepsilon^{D}$.

Optimal fit of the experimental data was found using two Tauc-Lorentz oscillators. 
Along the extraordinary axis, surface plasmons at the STO/LSTO interfaces couple to each other to form a collective oscillator that can be described by a Lorentz contribution ${ }^{5,6,7,8,9}$, wich was combined to a Tauc Lorentz contribution describing the dispersion at higher energy :

$\varepsilon_{e}=\varepsilon_{\infty}+\varepsilon^{L}+\varepsilon^{T L}$.

These functions provided excellent modeling of the ellipsometric experimental data, as shown in Fig.SI.7.

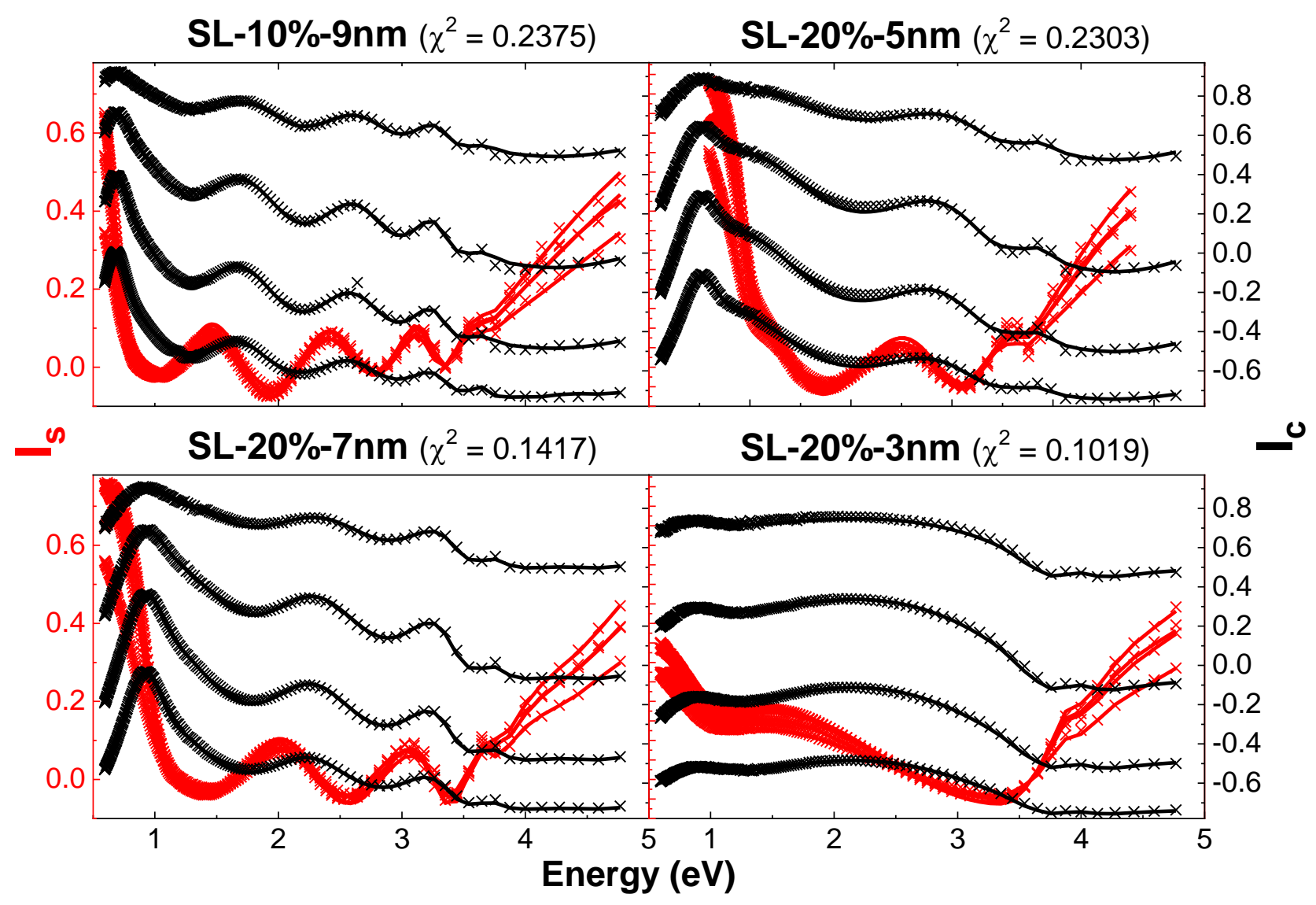

Fig.S7: Experimental (crosses) and modeled (solid lines) ellipsometric parameters for the superlattice samples (four incident angles $\theta=55,63,71$ and $79^{\circ}$ representative of the 13 used for the fitting procedure). $I_{\mathrm{s}}$ and $I_{\mathrm{c}}$ are related to the ellipsometric angles $\psi$ and $\Delta$ by $I_{s}=\sin (2 \psi) \times \sin (\Delta)$ and $I_{c}=$ $\sin (2 \psi) \times \cos (\Delta)$. Very low $\chi^{2}$ values attest for the excellent agreement between calculated and experimental ellipsometric data.

The corresponding permittivites are shown in Fig.SI.8, and the associated oscillator parameters and thicknesses are shown in Tab.SI.3. 


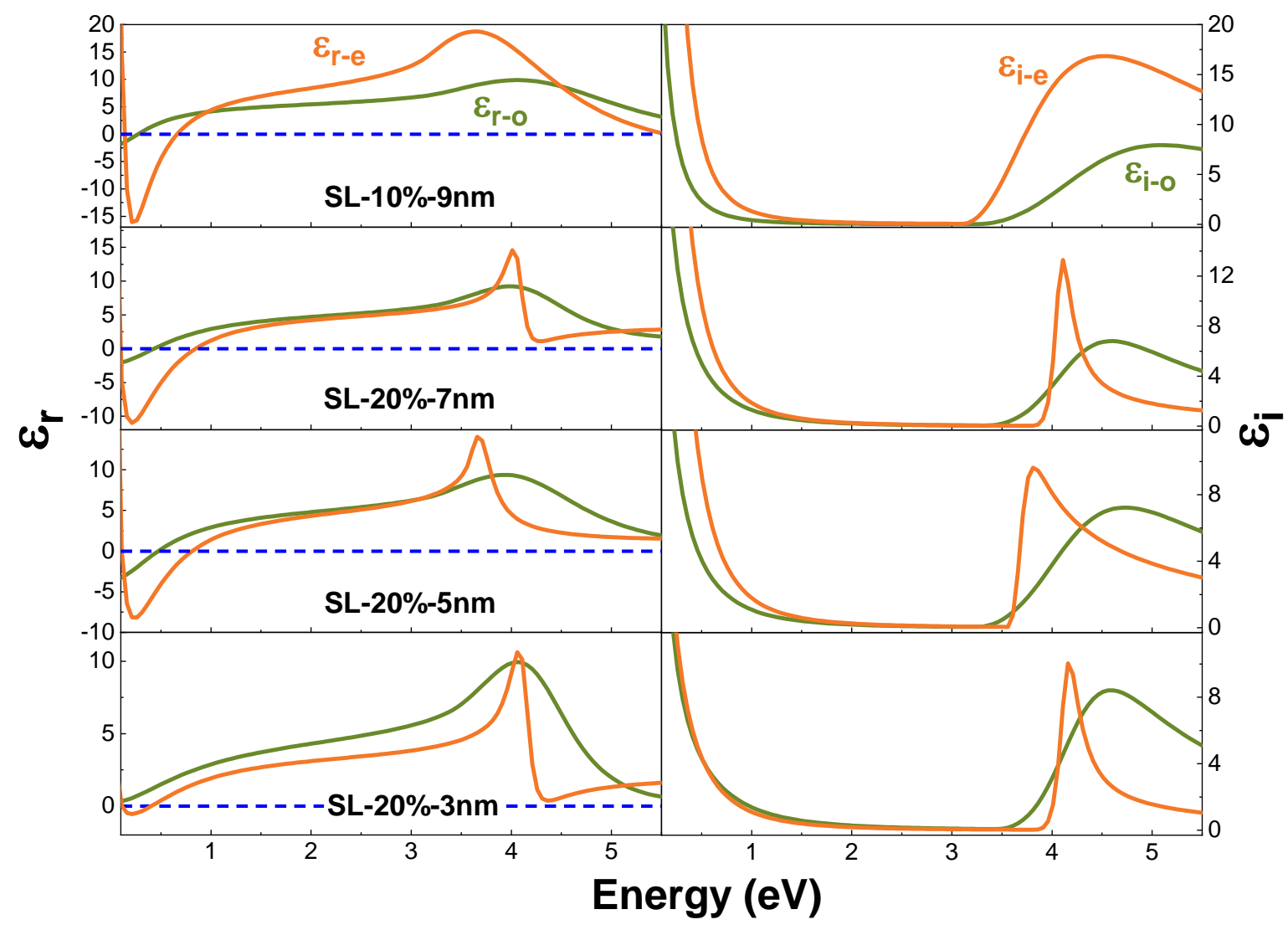

Fig.S8: Dielectric functions of the superlattice samples.

Ordinary component

\begin{tabular}{|c|c|c|c|c|c|c|c|c|c|c|}
\hline \multirow[b]{2}{*}{ Sample } & \multirow[b]{2}{*}{$\varepsilon_{\infty}$} & \multirow[b]{2}{*}{$\begin{array}{c}\mathbf{E g} \\
(\mathrm{eV})\end{array}$} & \multicolumn{3}{|c|}{ Tauc-Lorentz 1} & \multicolumn{3}{|c|}{ Tauc-Lorentz 2} & \multicolumn{2}{|c|}{ Drude } \\
\hline & & & $\begin{array}{c}\mathbf{A} \\
(\mathrm{eV})\end{array}$ & $\begin{array}{c}\mathbf{E}_{0} \\
(\mathrm{eV})\end{array}$ & $\begin{array}{c}\mathrm{C} \\
(\mathrm{eV})\end{array}$ & $\begin{array}{c}\mathbf{A} \\
(\mathbf{e V})\end{array}$ & $\begin{array}{c}E_{0} \\
(\mathrm{eV})\end{array}$ & $\begin{array}{c}\mathrm{C} \\
(\mathrm{eV})\end{array}$ & $\begin{array}{c}\omega_{\mathrm{p}} \\
(\mathrm{eV})\end{array}$ & $\begin{array}{c}\Gamma_{\mathrm{D}} \\
(\mathrm{eV})\end{array}$ \\
\hline SL-10\%-9nm & 1.85 & 3.24 & 226 & 4.43 & 2.71 & 4.66 & 1.35 & 2.15 & 1.09 & 0.41 \\
\hline SL-20\%-7nm & 2.86 & 3.33 & 177 & 4.23 & 1.51 & 1.64 & 1.53 & 5.08 & 1.65 & 0.74 \\
\hline SL-20\%-5nm & 2.24 & 3.24 & 207 & 4.19 & 2.02 & 9.70 & 2.00 & 9.22 & 1.50 & 0.77 \\
\hline SL-20\%-3nm & 2.07 & 3.44 & 230 & 4.27 & 1.32 & 2.24 & 2.50 & 11.4 & 1.66 & 1.04 \\
\hline
\end{tabular}

Extraordinary component

\begin{tabular}{|c|c|c|c|c|c|c|c|c|}
\hline \multirow[b]{2}{*}{ Sample } & \multirow[b]{2}{*}{$\varepsilon_{\infty}$} & \multirow[b]{2}{*}{$\begin{array}{c}\mathbf{E g} \\
(\mathbf{e V})\end{array}$} & \multicolumn{3}{|c|}{ Lorentz } & \multicolumn{3}{|c|}{ Tauc-Lorentz 1} \\
\hline & & & f & $\begin{array}{c}\omega_{0} \\
(\mathrm{eV})\end{array}$ & $\begin{array}{c}\Gamma_{\mathbf{L}} \\
(\mathrm{eV})\end{array}$ & $\begin{array}{c}\mathbf{A} \\
(\mathbf{e V})\end{array}$ & $\begin{array}{c}\mathrm{E}_{0} \\
(\mathrm{eV})\end{array}$ & $\begin{array}{c}\mathrm{C} \\
(\mathrm{eV})\end{array}$ \\
\hline SL-10\%-9nm & 0.51 & 3.10 & 310 & 0.118 & 0.320 & 687 & 3.66 & 2.12 \\
\hline SL-20\%-7nm & 3.97 & 3.80 & 740 & 0.080 & 0.471 & 546 & 4.07 & 0.20 \\
\hline SL-20\%-5nm & 2.63 & 3.56 & 502 & 0.094 & 0.495 & 1232 & 3.67 & 0.27 \\
\hline SL-20\%-3nm & 2.50 & 3.83 & 771 & 0.054 & 0.710 & 391 & 4.13 & 0.22 \\
\hline
\end{tabular}

Thicknesses

\begin{tabular}{|c|c|c|}
\hline Sample & Thickness $(\mathrm{nm})$ & Error $( \pm \mathrm{nm})$ \\
\hline SL-10\%-9nm & 248 & 1 \\
\hline SL-20\%-7nm & 199 & 2.1 \\
\hline SL-20\%-5nm & 144.2 & 0.6 \\
\hline SL-20\%-3nm & 61 & 1.7 \\
\hline
\end{tabular}

Tab.S3: oscillator parameters and thicknesses (deduced from ellipsometry measurements) for the superlattice samples 


\section{$\underline{\text { References }}$}

${ }^{1}$ Janotti, A.; Jalan, B.; Stemmer, S.; Van de Walle, C. G. Effects of Doping on the Lattice Parameter of SrTiO 3. Appl. Phys. Lett. 2012, 100 (26), 262104. https://doi.org/10.1063/1.4730998

${ }^{2}$ Apreutesei, M.; Debord, R.; Bouras, M.; Regreny, P.; Botella, C.; Benamrouche, A.; Carretero-Genevrier, A.; Gazquez, J.; Grenet, G.; Pailhès, S.; et al. Thermoelectric La-Doped SrTiO 3 Epitaxial Layers with Single-Crystal Quality: From Nano to Micrometers. Sci. Technol. Adv. Mater. 2017, 18 (1), 430-435. https://doi.org/10.1080/14686996.2017.1336055

${ }^{3}$ Jellison, G. E.; Modine, F. A. Parameterization of the Optical Functions of Amorphous Materials in the Interband Region. Appl. Phys. Lett. 1996, 69 (3), 371-373. https://doi.org/10.1063/1.118064.

${ }^{4}$ Zollner, S.; Demkov, A. A.; Liu, R.; Fejes, P. L.; Gregory, R. B.; Alluri, P.; Curless, J. A.; Yu, Z.; Ramdani, J.; Droopad, R.; et al. Optical Properties of Bulk and Thin-Film SrTiO[Sub 3] on Si and Pt. J. Vac. Sci. Technol. B Microelectron. Nanom. Struct. 2000, 18 (4), 2242. https://doi.org/10.1116/1.1303741.

${ }^{5}$ Camley, R. E.; Mills, D. L. Collective Excitations of Semi-Infinite Superlattice Structures: Surface Plasmons, Bulk Plasmons, and the Electron-Energy-Loss Spectrum. Phys. Rev. B 1984, 29 (4), 1695-1706. https://doi.org/10.1103/PhysRevB.29.1695.

${ }^{6}$ Yoshida, M.; Tomita, S.; Yanagi, H.; Hayashi, S. Resonant Photon Transport through Metal-Insulator-Metal Multilayers Consisting of Ag and $\mathrm{SiO}_{2}$. Phys. Rev. B 2010, 82 (4), 045410.

https://doi.org/10.1103/PhysRevB.82.045410.

${ }^{7}$ Naik, G. V.; Liu, J.; Kildishev, A. V.; Shalaev, V. M.; Boltasseva, A. Demonstration of Al:ZnO as a Plasmonic Component for near-Infrared Metamaterials. Proc. Natl. Acad. Sci. 2012, 109 (23), 8834-8838. https://doi.org/10.1073/pnas.1121517109

${ }^{8}$ Hoffman, A. J.; Alekseyev, L.; Howard, S. S.; Franz, K. J.; Wasserman, D.; Podolskiy, V. A.; Narimanov, E. E.; Sivco, D. L.; Gmachl, C. Negative Refraction in Semiconductor Metamaterials. Nat. Mater. 2007, 6 (12), $946-$ 950. https://doi.org/10.1038/nmat2033.

${ }^{9}$ Desouky, M.; Mahmoud, A. M.; Swillam, M. A. Silicon Based Mid-IR Super Absorber Using Hyperbolic Metamaterial. Sci. Rep. 2018, 8 (1), 2036. https://doi.org/10.1038/s41598-017-18737-5. 